Simulation gesellschaftlicher Medienwirkungsprozesse am Beispiel der Schweigespirale

by A. Waldherr and M. Bachl

To cite this article:

Waldherr, Annie \& Bachl, Marko (2011): Simulation gesellschaftlicher Medienwirkungsprozesse am Beispiel der Schweigespirale. In Suckfüll, Monika / Schramm, Holger \& Wünsch, Carsten (Eds.):

Rezeption und Wirkung in zeitlicher Perspektive (pp. 203-220). Baden-Baden: Nomos.

Published in:

M. Suckfüll / H. Schramm \& C. Wünsch (Eds.): Rezeption und Wirkung in zeitlicher Perspektive, BadenBaden: Nomos. (Link to the Book)

Copyright:

The Authors, 2011

DOI:

$10.5771 / 9783845231310$ 


\begin{abstract}
:
Der Beitrag stellt mit der agentenbasierten Modellierung (ABM) eine Methode zur Diskussion, mit der sich dynamische Medienwirkungsprozesse auf mehreren Ebenen modellieren und simulieren lassen. Dazu wird das Mikro-Makro-Problem in der Medienwirkungsforschung genauer erläutert und aus Sicht der Komplexitätstheorie interpretiert. Die Methode der Computersimulation sozialer Prozesse, speziell mittels ABM, wird erläutert. Schließlich wird die ABM am Beispiel der Schweigespirale vorgestellt, um ihre Eignung für die Untersuchung dynamischer, gesellschaftlicher Medienwirkungsprozesse zu demonstrieren. Hierzu werden die Annahmen der Schweigespirale nach Noelle-Neumann in einem Computermodell formalisiert und in ihrer Dynamik simuliert. Nach der Darstellung zentraler Simulationsergebnisse werden abschließend Chancen und Grenzen der Simulationsmethode für die Medienwirkungsforschung diskutiert.
\end{abstract}

\title{
Kurz-Biographien:
}

Bachl, Marko, M.A., Jg. 1983.

Studium Medien und Kommunikation (B.A.) an der Universität Augsburg und Medienmanagement (M.A.) am Institut für Journalistik und Kommunikationsforschung der Hochschule für Musik, Theater und Medien Hannover.

Seit 2008 wissenschaftlicher Mitarbeiter am Lehrstuhl für Kommunikationswissenschaft, insbesondere Kommunikationstheorie der Universität Hohenheim. Forschungsinteressen: Medienwirkungsforschung (v.a. in Politik und Wirtschaft), Politische Kommunikation sowie Methoden und Statistik in der Kommunikationswissenschaft.

Waldherr, Annie, Dipl. rer. com, Jg. 1980

Studium der Kommunikationswissenschaft an der Universität Hohenheim und an der University of Oregon (USA).

Von 2006 bis 2010 wissenschaftliche Mitarbeiterin am Lehrstuhl für Kommunikationswissenschaft, insbesondere Medienpolitik an der Universität Hohenheim. Seit Oktober 2010 wissenschaftliche Mitarbeiterin am Institut für Publizistik- und Kommunikationswissenschaft der Freien Universität Berlin. Forschungsschwerpunkte: Öffentlichkeit, Agenda-Building, Social Simulation, Technologie- und Wissenschaftsdiskurse, Interpersonale Kommunikation.

Annie Waldherr und Marko Bachl 


\section{Simulation gesellschaftlicher Medienwirkungsprozesse am Beispiel der Schweigespirale}

Medienwirkungen auf gesellschaftlicher Ebene sind häufig langfristig, kumulativ und empirisch schwer nachweisbar. Sie sind das Ergebnis dynamischer Prozesse und Interaktionen auf Mikro- und Mesoebene, und manifestieren sich in komplexen Wirkmustern auf der Makroebene. Wie genau die Prozesse auf den verschiedenen Ebenen miteinander verknüpft sind, ist in der Medienwirkungsforschung nach wie vor weitgehend ungeklärt. Scheufele (2008) spricht in diesem Zusammenhang vom „Erklärungsdilemma der Medienwirkungsforschung“ und einer empirischen Blackbox, die mit dem aktuellen empirischen Methodenrepertoire nicht zufrieden stellend geöffnet werden kann.

Ziel dieses Beitrages ist es, eine Methode zur Diskussion zu stellen, mit der sich dynamische Medienwirkungsprozesse auf mehreren Ebenen modellieren und simulieren lassen. Im ersten Abschnitt wird zunächst das Mikro-Makro-Problem in der Medienwirkungsforschung genauer erläutert und aus Sicht der Komplexitätstheorie interpretiert. Im zweiten Abschnitt wird die Methode der Computersimulation sozialer Prozesse vorgestellt, die neue Möglichkeiten zur Untersuchung von MikroMakro-Beziehungen bietet. Ein von Sozialwissenschaftlern immer häufiger verwendetes Simulationsinstrument, die Methode der agentenbasierten Modellierung (ABM), wird ebenfalls in diesem Abschnitt diskutiert.

Die Methode der ABM wird schließlich im dritten Abschnitt am Beispiel der Schweigespirale eingesetzt, um ihre Eignung für die Untersuchung dynamischer, gesellschaftlicher Medienwirkungsprozesse zu demonstrieren. Hierzu werden die Annahmen der Schweigespirale nach Noelle-Neumann in einem Computermodell formalisiert und in ihrer Dynamik simuliert. Dabei gehen wir in zwei Schritten vor. Das Grundmodell (Modell 1) beinhaltet nur individuelle Agenten, die miteinander nach den Regeln der Schweigespirale interagieren. Im erweiterten Modell (Modell 2) werden zusätzlich Massenmedien integriert. Nach der Darstellung zentraler Simulationsergebnisse werden im vierten Abschnitt Chancen und Grenzen der Simulationsmethode für die Medienwirkungsforschung diskutiert. 


\section{Das Mikro-Makro-Problem}

Die Erforschung der Zusammenhänge zwischen Mikro- und Makroebene, d.h. zwischen Individuen und gesellschaftlichen Strukturen, gehören zu den zentralen und herausfordernden Problemen in den Sozialwissenschaften. Medienwirkungsforschung, die sich nicht auf die Erforschung individueller Effekte von Massenmedienkonsum beschränken möchte, muss sich zwangsläufig mit dieser Problematik beschäftigen (Kepplinger, 1982, Scheufele, 2008).

Klassische Mikro-Makro-Probleme sind zum Beispiel aus der Agenda-SettingForschung bekannt (Schenk, 2007). Während Agenda-Setting-Effekte nur auf Aggregatebene relativ zuverlässig nachgewiesen wurden, sind die Ergebnisse auf Individualebene kaum existent. Die Frage ist, durch welche interaktive Dynamik die Prozesse auf Makroebene entstehen. Ein weiteres Beispiel ist das Phänomen der öffentlichen Meinung. Viele Forscher sind der Ansicht, dass öffentliche Meinung nicht nur durch aggregierte Einzelmeinungen beschrieben werden kann (Entman \& Herbst, 2001, Habermas, 1995, Noelle-Neumann, 1974). Die Dynamiken, die zur Herausbildung einer kollektiven öffentlichen Meinung führen, liegen jedoch noch weitgehend im Dunkeln.

Scheufele (2008) legt eindrücklich dar, auf welche Erklärungsschwierigkeiten Medienwirkungsforschung trifft, die nach dem statistischen Prinzip der Varianzzerlegung vorgeht. Ziel dieser Vorgehensweise ist es herauszufinden, wie groß der Anteil in der Varianz einer Variablen ist, der durch bestimmte Prädiktoren erklärt werden kann. Diese Vorgehensweise stößt an ihre Grenzen, wenn Theorien geprüft werden sollen, die Zusammenhänge zwischen verschiedenen Ebenen postulieren.

Das zentrale Problem sieht Scheufele (2008) allerdings darin, dass sich dynamische Mikro-Makro-Einflüsse methodisch-statistisch nur sehr schwer untersuchen lassen. Die Überprüfung von Brückenthesen über Makro-Mikro-Zusammenhänge und Aggregationsregeln über Mikro-Makro-Einflüsse (vgl. Esser, 1993) stelle Anforderungen an eine Menge und Qualität empirischer Daten, die zum Teil aus forschungspragmatischen, zum Teil aus logischen und zum Teil aus ethischen Gründen nicht realisierbar sind. Aus diesem Grund blieben theoretische Erklärungen in vielen Fällen reine „Trockenübungen“ (Scheufele, 2008, S. 350).

Das Prinzip der Varianzzerlegung stößt vor allem dann an seine Grenzen, wenn sich Faktoren auf Mikro- und Makro-Ebene in einem dynamischen Zusammenspiel gegenseitig bedingen (Scheufele, 2008, S. 351-353). Zusätzlich erschwert wird die empirische Analyse dadurch, dass Wirkungen häufig nichtlinear sind, Ursache und 
Wirkung also nicht unbedingt proportional zueinander sein müssen. Scheufele vermutet, dass sich diese Probleme methodisch-statistisch zum Teil gar nicht lösen ließen, vor allem da „eine verbesserte Erschließung und Verknüpfung von Medienwirkungen auf verschiedenen Ebenen mit einer verschlechterten Modellierung ihrer Dynamik einher" gehe (Scheufele, 2008, S. 355).

Die Probleme, die Scheufele (2008) anspricht, sind typische Merkmale komplexer Systeme. Besonders relevant sind bei komplexen Systemen die Beziehungen und Abhängigkeiten zwischen den einzelnen Komponenten. Schon die Löschung einer kritischen Komponente kann enorme Auswirkungen auf das Verhalten des gesamten Systems haben (Miller \& Page, 2007, S. 9, Richter \& Rost, 2004, S. 3). Dynamisches, nichtlineares Systemverhalten und Feedbackschleifen über verschiedene Ebenen hinweg gehören ebenfalls zu den Charakteristika komplexer Systeme (Capra, 1997, Jervis, 1997, Mainzer, 2007, Mitchell, 2009). Es wird hervorgerufen durch die Interaktionen verschiedener heterogener und adaptiver Agenten auf der Mikroebene. Diese Agenten sind in der Lage, Informationen aus der Umwelt zu verarbeiten und ihr Handeln daran anzupassen. In solchen Systemen entsteht Ordnung ohne zentrale Steuerung, indem die Agenten sich selbst organisieren (Holland, 1998). Dieser „Bottom-Up-Effekt" (Scheufele, 2008, S. 352), bei dem lokale Interaktionen auf der Mikroebene eine Regelmäßigkeit, ein Muster oder eine Eigenschaft auf Makroebene hervorbringen, kursiert in der Komplexitätsforschung unter dem Begriff der Emergenz (Holland, 1998, Sawyer, 2005). Eine Systemeigenschaft ist emergent, wenn das System als Ganzes diese Eigenschaft besitzt und keiner der Agenten allein (Bunge, 2003, S. 83).

In der sozialwissenschaftlichen Komplexitätsforschung werden Rückwirkungen von Makroeigenschaften auf die Mikroebene außerdem als Emergenz zweiter Ordnung beschrieben (Squazzoni, 2008, S. 6-7). Agenten bringen das Makrophänomen in diesem Fall nicht nur hervor, sondern erkennen es und reagieren darauf. Diese Form der Emergenz wird auch unter dem Begriff der Abwärtskausalität (,downward causation“) diskutiert (Conte, 2009, Sawyer, 2005). Scheufele (2008, S. 352) bezeichnet diesen Prozess als „Top-Down-Feedback“. Er ist ein wichtiges Charakteristikum sozialer Systeme, da hier davon auszugehen ist, dass die Agenten in der Lage sind, über Makrostrukturen des Systems zu reflektieren. 


\section{Simulation dynamischer sozialer Prozesse}

Die Tatsache, dass sich nicht alle relevanten Prozesse in gesellschaftlichen Systemen empirisch lückenlos beobachten und erfassen lassen, brachte Sozialwissenschaftler im vergangenen Jahrzehnt zunehmend dazu, die theoretischen „Trockenübungen" durch Computersimulationen anzureichern. Die interessierenden Prozesse werden in dynamischen Computermodellen nachgebildet. Hiermit lassen sich virtuelle Experimente durchführen, die in der Realität so nicht möglich sind. In wenigen Minuten lassen sich am Computer tausende von Zeitschritten simulieren. Dies macht es möglich, Szenarien mit unterschiedlichen Anfangsbedingungen durchzuspielen und ihre langfristigen Wirkungen zu beobachten. Statt des realen Systemverhaltens wird also das Modellverhalten beobachtet (Gilbert \& Troitzsch, 2005).

Epstein (2006) nennt diesen Zugang ,generative science“. Ziel ist, komplexe Makrostrukturen in der Simulation durch möglichst einfache lokale Interaktionen der Agenten auf der Mikroebene zu generieren. Auf diese Weise kann eine Reihe hinreichender Mikrobedingungen spezifiziert werden, die in der Lage sind, ein bestimmtes Muster auf Makroebene hervorzurufen. Gelingt dies, ist ein wichtiger Schritt zur Erklärung eines Makrophänomens gelungen. Allerdings müssen diese Bedingungen nicht unbedingt den Bedingungen in der Realität entsprechen (Miller \& Page, 2007, S. 86). Dieser Vorbehalt wird besonders deutlich, wenn unterschiedliche Realisierungen von Mikrobedingungen zum gleichen kollektiven Systemverhalten führen (Conte, 2009, Squazzoni, 2008). Daher ist es wichtig, ein in sich kohärentes Simulationsmodell immer wieder mit der Realität zu konfrontieren (Boero \& Squazzoni, 2005, Windrum et al., 2007). Hierzu können aus dem Computermodell Hypothesen abgeleitet werden, die dann einzeln empirisch überprüft werden. Es wird jedoch meist nicht möglich sein, das gesamte Modell empirisch zu testen, denn dann wäre auch die Notwendigkeit für ein solches Modell nicht gegeben.

Ein Instrument, das sich gut zur Modellierung komplexer Systeme eignet und auch zunehmend von Sozialwissenschaftlern eingesetzt wird, ist die Agentenbasierte Modellierung (ABM). Agentenbasierte Modelle sind prozessorientierte Computermodelle. Sie bestehen aus folgenden Komponenten (Gilbert, 2007, S. 4 ff.):

- Agenten: Dies sind relativ autonome Softwareobjekte mit definierten Attributen und Zielen. Sie können Information aus der Umgebung oder von anderen Agenten aufnehmen und ihre Handlungen an diese Informationen anpassen. 
- Umwelt: Die Agenten interagieren in einer virtuellen Umwelt. Dies kann ein topographischer Raum sein, ein Netzwerk oder auch ein abstrakter Wissensraum.

- Regeln: Agenten verarbeiten Informationen aus ihrer Umwelt und wählen ihre Aktionen gemäß definierter Regeln.

Abbildung 1 zeigt, wie sich die Vorgehensweise der Computersimulation in das Modell der soziologischen Erklärung (vgl. Esser, 1993) integrieren lässt. Die Agenten nehmen Informationen aus ihrer Umwelt gemäß definierter Wahrnehmungsregeln auf. Die Situationslogik wird im Modell also durch diese Regeln definiert. Ebenfalls spezifiziert werden die Regeln der Selektionslogik, nach denen die Agenten ihre Handlungen auswählen. Dazu werden Wenn-Dann-Bedingungen formuliert. Die Makromuster entstehen in der Simulation aus den Interaktionen der Agenten heraus. Das bedeutet für die Aggregationslogik, dass keine spezifischen Regeln implementiert werden. Die kollektiven Muster entstehen durch simulierte Emergenz.

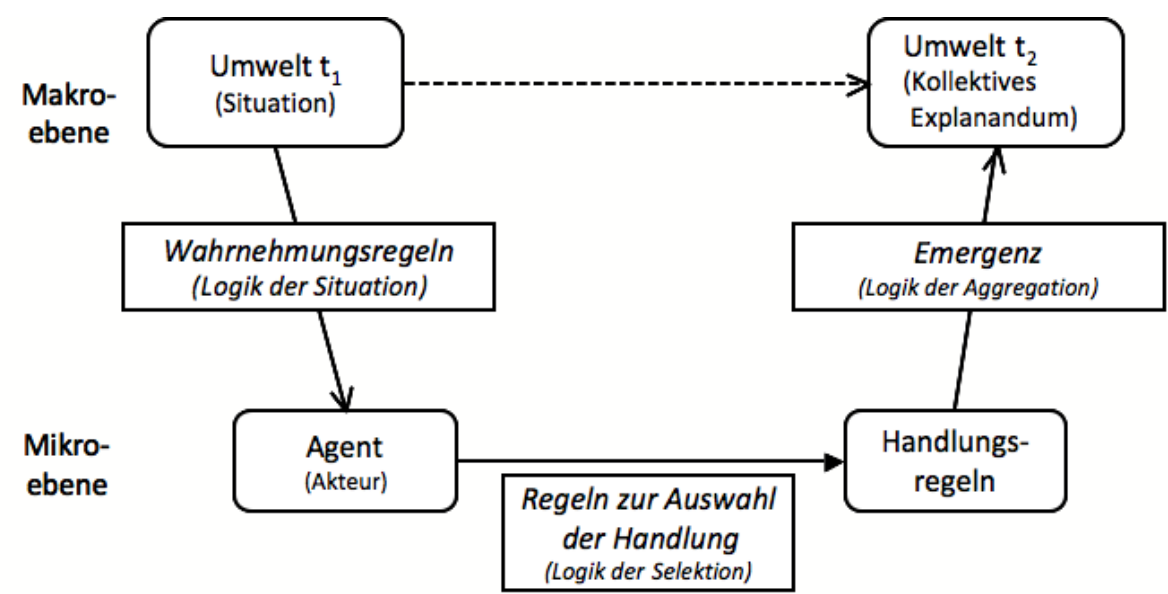

Anmerkung: Die Begriffe in Klammern entsprechen dem sogenannten Badewannenmodell der soziologischen Erklärung nach Esser (1993).

\section{Abbildung 1: Die Logik der Simulation sozialer Prozesse}


Mit der Möglichkeit, heterogene, adaptive, interagierende Agenten abzubilden, eignet sich die ABM gut zur Modellierung komplexer sozialer Systeme (Miller \& Page, 2007, S. 78-89). Zum einen bieten sie einen guten Kompromiss zwischen Flexibilität und Präzision, da die Formalisierung in Computersprache zu mehr Präzision zwingt als rein sprachlich formulierte Modelle, jedoch nicht so hohe analytische Anforderungen stellt wie mathematische Modelle. Zum anderen sind ABM stark prozessorientiert und bieten eine geeignete Technik zur Modellierung dynamischer Prozesse. Die Modelle sind beliebig skalierbar und die Simulationen beliebig oft wiederholbar. Dies ermöglicht Computerexperimente, die in der Realität auf diese Weise nicht möglich sind.

In der Kommunikationswissenschaft allgemein und in der Medienwirkungsforschung im Besonderen wird ABM bisher jedoch kaum eingesetzt. Eine vergleichsweise rege Forschungstätigkeit lässt sich auf dem Gebiet der Meinungsdynamik feststellen (Gilbert, 2007, S. 8-9). Die Forscher sind jedoch meist keine Kommunikationswissenschaftler, sondern stammen aus anderen Disziplinen, etwa aus der Psychologie, Philosophie oder Physik. Anhand von Netzwerkmodellen wird zum Beispiel simuliert, wie sich Meinungen in einer Population von Agenten verbreiten und durchsetzen (Deffuant et al., 2002, González-Avella et al., 2007, Hegselmann \& Krause, 2002, McKeown \& Sheehy, 2006). Die Rolle der Massenmedien wird in der bisherigen Forschung jedoch bis auf einzelne Ausnahmen (Gong \& Xiao, 2007, McKeown \& Sheehy, 2006) vernachlässigt.

\section{Ein agentenbasiertes Modell der Schweigespirale}

Am Beispiel der Schweigespirale (Noelle-Neumann, 1974) diskutieren wir das Potenzial der ABM für die Untersuchung von Medienwirkungen auf gesellschaftlicher Ebene. Die Theorie eignet sich als Anwendungsbeispiel besonders, da sie sowohl Aussagen über das Verhalten der einzelnen Individuen als auch über dessen Folgen für das gesellschaftliche Meinungsklima trifft. Diese Integration von Mikround Makro-Ebene wird in theoretischer Hinsicht als lobenswert herausgestellt, führt aber zu Schwierigkeiten bei der empirischen Überprüfung (Donsbach \& Stevenson, 1986, Schenk, 2007, S. 532 f.). Problematisch ist unter anderem der immense Aufwand langfristiger Panel-Untersuchungen, die über genügend Messzeitpunkte verfügen, um die Dynamiken abzubilden (z.B. Scherer, 1990). Eine Überprüfung im Sinne eines empirischen Tests kann auch ein ABM nicht leisten. Die Methode kann allerdings dabei helfen, den Zusammenhang zwischen Veränderungen im individuel- 
len Verhalten und der öffentlichen Meinung transparent zu machen, die Thesen der Schweigespirale auf ihre interne Konsistenz zu prüfen und weitere Hypothesen über das Zusammenspiel von Mikro- und Makroebene zu entwickeln.

Bisher gibt es u.W. noch kein agentenbasiertes Modell der Schweigespirale. Granovetter und Soong (1988) sowie Krassa (1988) haben die Theorie mathematisch modelliert und auf dieser Basis Simulationen durchgeführt. Die mathematischen Modelle bewegen sich jedoch hauptsächlich auf der Makroebene. Dadurch lässt sich die Heterogenität und Vernetzung der sozialen Agenten nur sehr begrenzt abbilden. Die Forscher konzentrierten sich vor allem auf unterschiedliche Verteilungen von Handlungsschwellen in der Population. Die Wirkung der Massenmedien auf den Prozess der Schweigespirale wurde in diesen Modellen nicht untersucht.

Krassa (1988) führte zusätzlich Netzwerke, soziale Gruppen und Meinungsführer ein. Dadurch wurden die Differentialgleichungen aber so komplex, dass sie nicht mehr analytisch lösbar waren und Computersimulationen nötig wurden. Hier hat die ABM eindeutige Vorteile, da die Agenten auf Mikroebene modelliert werden.

Die Annahmen der Schweigespirale sind bereits sehr präzise ausformuliert und bieten eine gute Vorlage für die Konstruktion eines ABM. Noelle-Neumann (1993) formulierte drei zentrale Hypothesen zum Verhalten von Individuen (vgl. zusammenfassend Schenk, 2007, S. 533):

- Quasi-statistische Wahrnehmung: Individuen nehmen kontinuierlich wahr, welche unter verschiedenen widersprüchlichen Meinungen die Mehrheitsmeinung ist.

- Isolationsfurcht: Individuen verspüren die Furcht, sich durch gesellschaftlich nicht akzeptierte Äußerungen zu isolieren.

- Redebereitschaft: Individuen, die ihre Meinung in der Gesellschaft in ausreichendem Maße vertreten sehen, äußern ihre eigene Meinung mit größerer Wahrscheinlichkeit.

Diese Hypothesen bilden die grundlegenden Regeln auf Mikroebene, nach denen die Agenten handeln. Die Annahmen werden in zwei Schritten in ein ABM implementiert. Zunächst wird auf Basis der genannten Individualhypothesen ein Grundmodell der Schweigespirale ohne Massenmedien modelliert (Modell 1). Unter der Voraussetzung, dass auf dieser Grundlage ein theoriekonformes System geschaffen werden kann, werden in einem zweiten Schritt Massenmedien als weitere Agenten eingeführt, um Noelle-Neumanns Annahme einer Beeinflussung des Meinungsklimas durch die publizierte Meinung im Modell zu berücksichtigen (Modell 2). Durch 
die schrittweise Erweiterung kann beobachtet werden, inwiefern sich das Modellverhalten durch die Einführung der Massenmedien verändert.

\section{Modell ohne Massenmedien (Modell 1)}

Im ersten Schritt entwerfen wir ein grundlegendes Modell, das die drei zentralen Hypothesen der Schweigespirale auf Individualebene berücksichtigt. Ziel ist, ein System zu schaffen, das auf Makroebene die erwarteten Muster und Dynamiken zeigt. Bei einer anfänglich ausgeglichenen Meinungsverteilung sollen beide Meinungen dauerhaft in der Öffentlichkeit präsent sein. Dominiert hingegen eine Meinung zu Beginn der Simulation deutlich, soll es zu einer Schweigespiralen-Dynamik kommen, die dazu führt, dass Vertreter der Minderheitsmeinung verstummen.

Umwelt, Agenten und Attribute: Die Agenten werden zu Beginn der Simulation zufällig in einer virtuellen Umwelt verteilt, die in quadratische Felder (sog. ,Patches $^{`}$ ) gleicher Fläche aufgeteilt ist. Die Felder repräsentieren das Wahrnehmungsfeld der Agenten. Jeder Agent kann mit den Agenten auf dem eigenen Feld und den acht benachbarten Feldern interagieren.

Modell 1 enthält zwei Klassen individueller Agenten (vgl. Abbildung 2). Jeder Agent besitzt folgende Attribute:

- Eine Einschätzung des Meinungsklimas $\mathrm{MK}_{\mathrm{X}}$, ausgedrückt als Anteil der eigenen Meinung an allen wahrgenommenen Meinungen. Der Startwert für alle Agenten ist 1. Während der Simulation wird der Wert in jedem Zeitschritt aktualisiert und liegt dann zwischen 0 und 1.

- Eine Isolationsfurchtschwelle IF, ab der der Agent annimmt, dass die Meinungsäußerung zu Isolation führt. IF liegt im Sinne einer einfachen Interpretation von „Mehrheit“ bei 0,5 und ist sowohl über die Zeit als auch über alle Agenten konstant.

- Eine Redebereitschaft $\left(\mathrm{RB}_{\mathrm{x}}\right)$ mit den dichotomen Ausprägungen $0=$ keine Meinungsäußerung und 1 = Meinungsäußerung. Der Startwert für alle Agenten ist 1: Dadurch wird eine Initialeinstellung realisiert, in der sämtliche Agenten bereit sind, ihre Meinung zu äußern. Alternativ wären auch hier variable Startbedingungen möglich. Während der Simulation wird der Wert in jedem Zeitschritt neu ermittelt, wodurch sich dynamische Prozesse im Meinungsklima ergeben. 


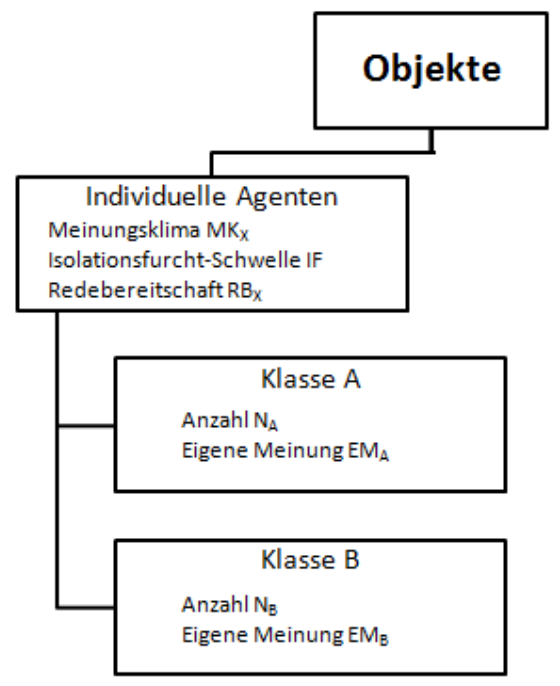

Abbildung 2: Objekte und Attribute in Modell 1

Die Agenten der Klassen A und B repräsentieren Gruppen von Individuen mit zwei unterschiedlichen eigenen Meinungen $\mathrm{EM}_{\mathrm{A}}$ und $\mathrm{EM}_{\mathrm{B}}$. Die Meinungen bleiben für alle Agenten über die Zeit konstant. Zu Beginn jeder Simulation wird festgelegt, wie viele Agenten $\mathrm{N}_{\mathrm{A}}$ und $\mathrm{N}_{\mathrm{B}}$ der beiden Klassen in der Umwelt existieren sollen. Durch das Verhältnis der beiden Klassen werden Mehrheits- und Minderheitsmeinungen dargestellt. Die Gesamtzahl der Agenten beeinflusst (bei unveränderter Größe der Umwelt) die Interaktionsdichte des Systems. Je mehr Agenten sich im gesamten System befinden, desto mehr Agenten sind auch im direkten Umfeld jedes Agenten und werden bei Interaktionen einbezogen. Da sich die konkrete Kontaktzahl pro Zeitschritt nicht theoretisch oder empirisch ableiten lässt, werden in den Simulationen verschiedene plausible Werte für die Gesamtzahl der Agenten getestet.

Regeln und Ablauf der Simulation: (1) Zunächst bewegen sich alle Agenten in der virtuellen Umwelt. (2) Dann ermitteln die Agenten jeweils das aktuelle Meinungsklima in ihrem Umfeld. Dabei werden nur diejenigen Agenten berücksichtigt, die ihre Meinung gerade äußern. (3) Anschließend verrechnen die Agenten das aktuell wahrgenommene Meinungsklima mit ihren Erfahrungen der Vergangenheit. Dabei wird die vergangene Erfahrung mit einem Stabilitätsfaktor S gewichtet. In den hier präsentierten Modellen ist $S=0,9$, d.h. 90 Prozent der aktuellen Einschätzung 
des Meinungsklimas setzt sich aus Erfahrungen aus der Vergangenheit zusammen. ${ }^{1}$ Dies trägt dem Umstand Rechnung, dass sich die Einschätzung nicht von einem Augenblick zum nächsten völlig ändert, sondern sich im Sinne kumulierter Wirkungen langsam aktualisiert. (4) Schließlich gleicht jeder Agent seine Einschätzung des Meinungsklimas mit seiner Isolationsfurchtschwelle ab. Nimmt er seine Meinung als Minderheitsmeinung wahr, schweigt er. Sieht er sich auf Seiten der Mehrheit, äußert er seine Meinung. Tabelle 1 fasst den Ablauf der Agentenhandlungen formalisiert zusammen.

In der Simulation werden beliebig viele Wiederholungen dieser Prozeduren durchgeführt. Durch die ständige Veränderung der Agentenpositionen in der Umwelt (Prozedur 1) und die angepasste Redebereitschaft (Prozedur 4) ergibt sich ein dynamischer Wandel der wahrnehmbaren Meinungsverteilung im System. Da die Agenten die neuen Meinungsverteilungen wiederum wahrnehmen (Prozedur 2) und darauf reagieren (Prozeduren 3 und 4), folgt eine Rückkopplung auf die Individualebene. Zur Implementierung des ABM wurde die Software NetLogo 4.1.1 verwendet. $^{2}$

Tabelle 1: Ablauf der Prozeduren eines Zeitschritts (Modell 1)

\begin{tabular}{|c|c|}
\hline 1 & Bewegen in der Umwelt \\
\hline 2 & $\begin{array}{l}\text { Quasi-statistische Wahrnehmung des Meinungsklimas im Umfeld } \\
\text { Klasse } A: \mathrm{MK}_{\mathrm{x}}(\mathrm{t})=\text { Anzahl }\left(\text { Agenten } \mathrm{EM}_{\mathrm{A}} \text { mit } \mathrm{RB}_{\mathrm{x}}=1 \mathrm{im} \text { Umfeld) } / \text { Anzahl (Agenten mit } \mathrm{RB}_{\mathrm{x}}\right. \\
=1 \mathrm{im} \text { Umfeld) } \\
\text { Klasse } \mathrm{B}: \mathrm{MK}_{\mathrm{x}}(\mathrm{t})=\text { Anzahl (Agenten } \mathrm{EM}_{\mathrm{B}} \text { mit } \mathrm{RB}_{\mathrm{x}}=1 \mathrm{im} \text { Umfeld) } / \text { Anzahl (Agenten mit } \mathrm{RB}_{\mathrm{x}} \\
=1 \mathrm{im} \text { Umfeld) }\end{array}$ \\
\hline 3 & $\begin{array}{l}\text { Gewichtung vergangener Erfahrungen } \\
M K_{x}=M K_{x}(t) *(1-S)+M K_{x}(t-1) * S\end{array}$ \\
\hline 4 & $\begin{array}{l}\text { Ermitteln der Redebereitschaft aus Einschätzung des Meinungsklimas und Schwelle der } \\
\text { Isolationsfurcht } \\
\text { Wenn } \mathrm{MK}_{\mathrm{x}} \geq \mathrm{IF} \text {, dann } \mathrm{RB}_{\mathrm{x}}=1 \\
\text { Wenn } \mathrm{MK}_{\mathrm{x}} \leq \mathrm{IF} \text {, dann } \mathrm{RB}_{\mathrm{x}}=0\end{array}$ \\
\hline
\end{tabular}

\footnotetext{
${ }^{1}$ Der konkrete Wert von 0,9 wurde aus einer Reihe von Simulationsergebnissen mit Werten zwischen 0,99 und 0,7 ausgewählt. Das grundlegende Muster der Meinungsdynamiken ist innerhalb dieser Spannweite vergleichbar, Unterschiede ergeben sich in der Geschwindigkeit der Dynamiken. Auf eine ausführlichere Diskussion dieser Ergebnisse muss hier aus Platzgründen verzichtet werden.

${ }^{2}$ Die Software ist unter http://ccl.northwestern.edu/netlogo/ frei verfügbar.
} 


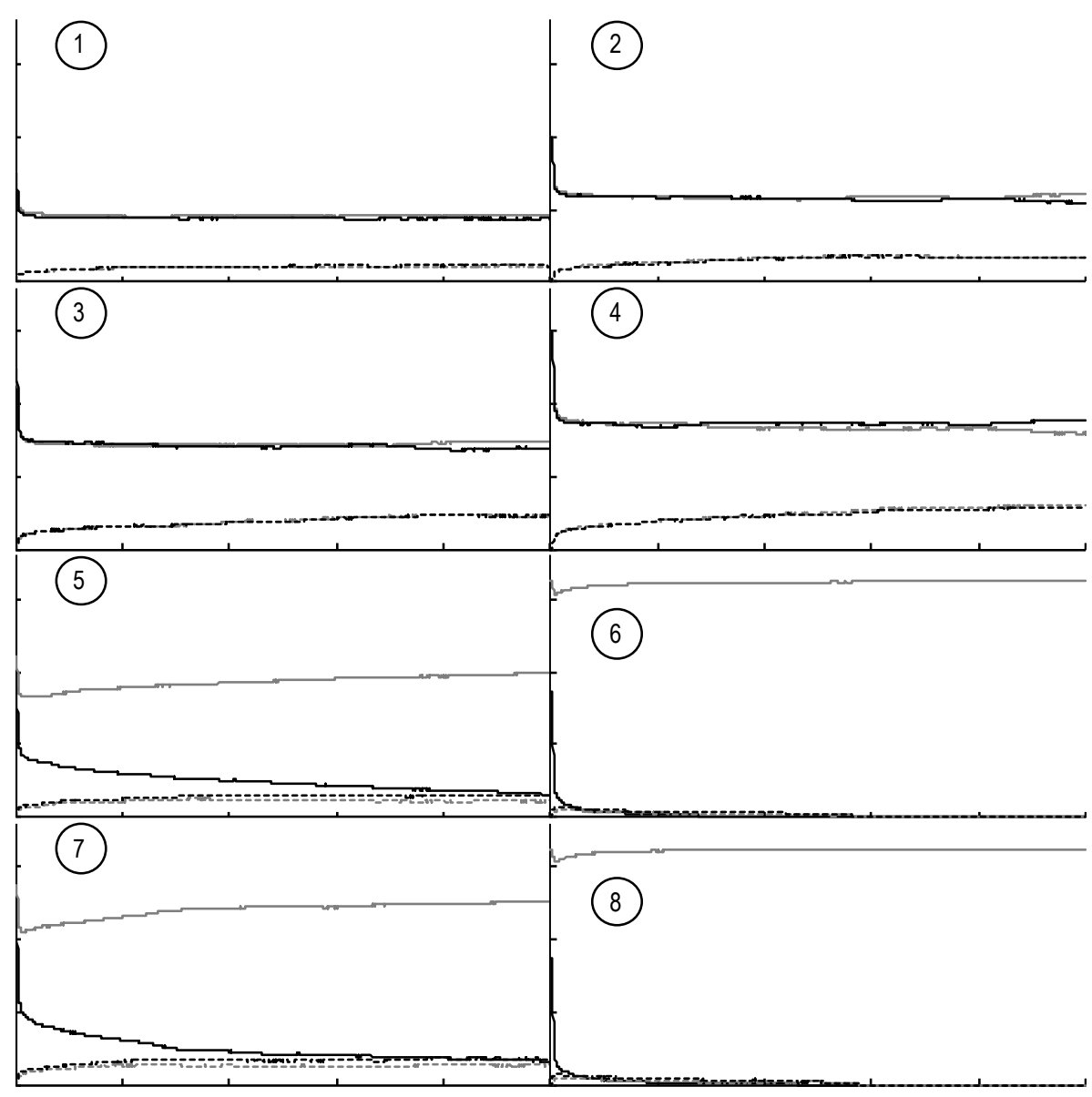

Anmerkungen: Durchgezogene Linien: Mittlere Anzahl der Agenten A und B mit $\mathrm{RB}_{\mathrm{X}}=1$. Gestrichelte Linien: Standardabweichung der Anzahl der Agenten mit $\mathrm{RB}_{\mathrm{X}}=1$. Jeweils 100 Simulationen mit 2500 Zeitschritten: 1) 150 Agenten, davon 50\% Klasse A; 2) 200, 50\%; 3) $250,50 \%$ 4) $300,50 \%$; 5) 200, 55\% ; 6) 200, 65\% ; 7) 250, 55\% ; 8) $250,65 \%$.

Abbildung 3: Ergebnisse der Simulation von Modell 1 bei ausgeglichener und unterschiedlicher Verteilung der Klassen A und B 
Ergebnisse der Simulationen: In wiederholten Simulationen überprüfen wir, ob sich durch die Spezifikationen auf Mikroebene die theoretisch postulierten Muster auf Makroebene ergeben. Zuerst testen wir Modelle mit einer ausgeglichenen Verteilung der Klassen A und B, wobei wir die Anzahl der Agenten variieren (vgl. Abbildung 3). Die Diagramme zeigen den Verlauf der Anzahl der Agenten beider Klassen, die zum jeweiligen Zeitschritt bereit waren, ihre Meinung zu äußern. Die Linien stehen dabei jeweils für den Mittelwert und die Standardabweichung aus 100 Simulationen, die mit unveränderter Konfiguration durchlaufen wurden. Durch die wiederholte Simulation wird der zufällige Einfluss, den die Startverteilung und die Bewegungen der Agenten auf das Modell ausüben, berücksichtigt. Jede Simulation wurde über 2500 Zeitschritte durchgeführt.

Die Ergebnisse zeigen, dass die theoretisch postulierten Entwicklungen auf Makroebene zu beobachten sind. Bei einer ausgeglichenen Verteilung der Klassen A und B (Abbildung 3, Spezifikationen 1-4) tritt keine Schweigespiral-Dynamik ein. Stattdessen sind die Vertreter beider Meinungen im Mittel über alle Durchläufe gleichermaßen redebereit. Die Verteilung zu einem Zeitpunkt schwankt zwar zwischen den Durchläufen recht deutlich, keine der Klassen kann das System aber dauerhaft innerhalb einer Simulation oder im Mittel über alle Simulationen dominieren.

Auch die Modellspezifikationen mit Mehr- und Minderheitsmeinungen zeigen die zu erwartenden Makromuster (Abbildung 3, Spezifikationen 5-8). Wenn die Agenten einer Klasse (hier Klasse A) die Mehrheit der Gesamtpopulation ausmachen, so wird ihr Anteil an den Agenten, die bereit sind, ihre Meinung zu äußern, noch größer eingeschätzt. Je größer die Mehrheit einer Klasse ist, desto häufiger kommt es zu einer Schweigespirale, an deren Ende die Vertreter der Minderheitsmeinung völlig verstummen.

Die Variation der Gesamtzahl der Agenten ergibt zudem, dass eine größere Kontakthäufigkeit zu einem schnelleren Verlauf der Schweigespiral-Dynamik und häufiger zu einem völligen Verstummen der Minderheit führt. Damit ist das erste Ziel der Modellentwicklung erreicht: Die Simulationen des auf Mikroebene spezifizierten ABM führen zu den theoretisch erwarteten Mustern auf der Makroebene.

Modell mit Massenmedien (Modell 2)

In einem zweiten Schritt führen wir nun weitere Agenten MA und MB mit den Meinungen $\mathrm{EM}_{\mathrm{A}}$ und $\mathrm{EM}_{\mathrm{B}}$ in das Modell ein, die die massenmedial verbreiteten Meinungen repräsentieren. Sie unterscheiden sich von den Agenten A und B insofern, dass ihre Meinung immer zu erkennen ist $(\mathrm{RB}=1)$. Die Regeln für die Agenten 
werden so erweitert, dass zusätzlich zu A und B auch MA und MB zur Einschätzung des Meinungsklimas im Umfeld herangezogen werden. Über die Veränderung des Verhältnisses von $\mathrm{N}_{\mathrm{MA}}$ und $\mathrm{N}_{\mathrm{MB}}$ kann nun getestet werden, welchen Einfluss der Medientenor auf die Meinungsdynamiken im System hat. ${ }^{3}$ Uns interessiert dabei besonders die Frage, ob der Medientenor das Potenzial hat, den Prozess der Schweigespirale umzukehren, was eine der Kernhypothesen von Noelle-Neumanns (1993) Theorie ist. Welche Bedingungen führen zu einer Situation, in der Vertreter der Mehrheitsmeinung schweigen, während die vom Medientenor unterstützten Vertreter der Minderheitsposition ihre Meinung weiter äußern?

Über alle Modellspezifikationen werden $\mathrm{N}_{\mathrm{A}}$ und $\mathrm{N}_{\mathrm{B}}$ konstant gehalten (vgl. Abbildung 4). Dabei macht $\mathrm{N}_{\mathrm{A}} 65$ Prozent der Gesamtpopulation aus und stellt damit die Mehrheitsmeinung - diese Konfiguration zeigt in den Simulationen ohne Massenmedien klare Schweigespiralen (vgl. Abbildung 3, Spezifikation 8). Das Medienklima, also das Verhältnis von $\mathrm{N}_{\mathrm{MA}}$ und $\mathrm{N}_{\mathrm{MB}}$, wird von Spezifikation zu Spezifikation in Richtung der Minderheitsmeinung B verändert. In Spezifikation 1 gehören 65 Prozent der massenmedialen Agenten der Klasse MB an, in Spezifikation 8 existieren ausschließlich mediale Agenten der Klasse MB. Damit repräsentiert diese letzte Modellannahme den Extremfall, dass in den Medien konsonant nur die Meinung der Minderheit präsent ist.

Die Ergebnisse zeigen, dass eine Überrepräsentation der Minderheitsmeinung in den Medien zu einer verminderten Redebereitschaft bei den Vertretern der eigentlichen Mehrheitsmeinung führen kann. Damit sich das gesamtgesellschaftliche Meinungsklima aber dahingehend ändert, dass die eigentliche Minderheitsmeinung als Majorität wahrgenommen wird, scheint eine sehr deutliche Dominanz dieser Meinung im Medienklima nötig zu sein. Erst in Spezifikation 5, in der nur 15 Prozent der medialen Informationen der Mehrheitsmeinung entsprechen, sind auf Dauer mehr Vertreter der Minderheitsmeinung als der Mehrheitsmeinung dazu bereit, ihre Meinung zu äußern. Eine schweigende Mehrheit (d.h. kein Vertreter der Mehrheitsmeinung äußert seine Meinung) ist erst bei einem konsonanten Medientenor, wie er in Spezifikation 8 angenommen wird, mit großer Sicherheit (99 von 100 Simulationen) wahrscheinlich. Selbst wenn die Mehrheitsmeinung nur fünf Prozent aller massenmedialen Informationen im System ausmacht (Spezifikation 7), ist nur in etwa der Hälfte der Fälle (53 von 100 Simulationen) zu einem Zeitschritt kein Vertreter dieser Meinung redebereit. Die Ergebnisse weisen damit auf die Bedeutung hin, die

\footnotetext{
${ }^{3}$ Darüber hinaus bleiben die Modellspezifikationen unverändert.
} 
auch Noelle-Neumann (1973) einem konsonanten Medienklima als Voraussetzung für gesellschaftliche Medienwirkungen zuschreibt.

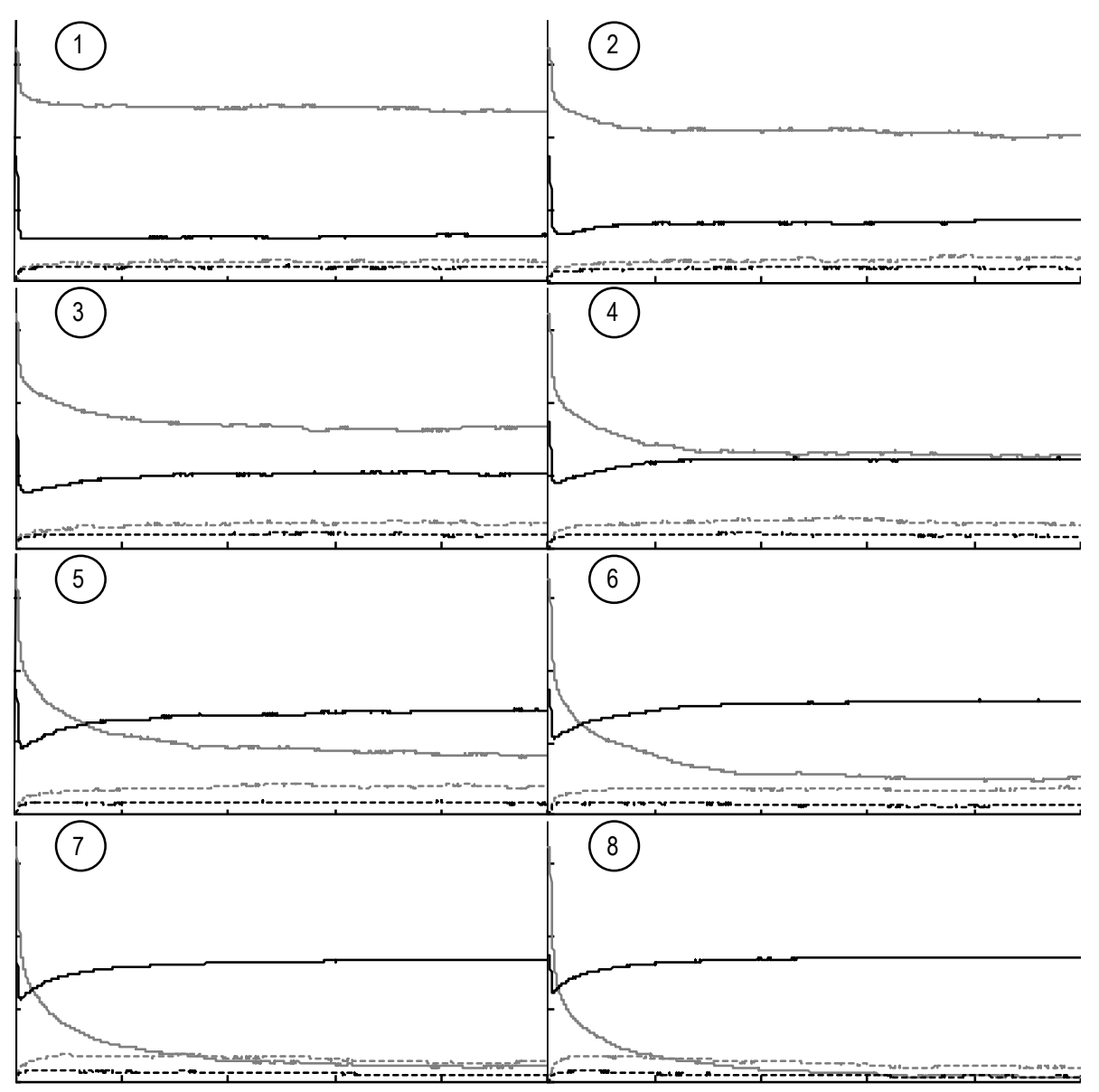

Anmerkungen: Durchgezogene Linien: Mittlere Anzahl der Agenten A und B mit $\mathrm{RB}_{\mathrm{X}}=1$. Gestrichelte Linien: Standardabweichung der Anzahl der Agenten mit $\mathrm{RB}_{\mathrm{X}}=1$. Jeweils 250 Agenten A und B, davon 65\% Klasse A (vgl. Abbildung 3: Ergebnisse der Simulation von Modell 1 bei ausgeglichener und unterschiedlicher Verteilung der Klassen A und B, 8); 100 Simulationen mit 2500 Zeitschritten: 1) 75 Agenten MA und MB, davon 35\% Klasse MA; 2) $75,30 \%$; 3) 75, 25\% ; 4) 75, 20\% ; 5) 75, 15\% ; 6)75, 10\% ;) 75, 5\% ; 8) 75, 0\% ; 
Abbildung 4: Ergebnisse der Simulation von Modell 2 bei einem der Mehrheitsmeinung gegenläufigen Medientenor 
Insgesamt zeigen die hier präsentierten Modelle, dass es möglich ist, die Theorie der Schweigespirale in ein ABM zu überführen. Dabei reichen wenige Spezifikationen auf Mikroebene aus, um die postulierten Dynamiken auf Makroebene zu simulieren. Bereits in diesen einfachen Modellen deuten sich einige interessante Erkenntnisse an. So zeigen sich z.B. Einflüsse der Interaktionsdichte, die sich durch die Anzahl der Agenten in der Umwelt variieren lässt. Diese Makrovariable wurde in der bisherigen Forschung kaum berücksichtigt, scheint jedoch Auswirkungen auf die zeitliche Dynamik der Prozesse auf Mikro- wie auf Makroebene zu haben.

Zudem weisen die Ergebnisse auf die große Bedeutung der Konsonanz in der Medienberichterstattung hin, die auch Noelle-Neumann (1973) bereits als zentrale Voraussetzung gesellschaftlicher Medienwirkungen anführte. Erstaunlich ist jedoch, wie stark die Konsonanz in den Simulationen ausgeprägt sein muss, um einen Schweigespiralprozess auszulösen. Erst wenn eine Meinung fast vollständig aus der publizierten Meinung verschwindet, verstummen auch die Anhänger dieser Meinung dauerhaft in der Öffentlichkeit.

Die hier vorgestellten Modelle sind lediglich ein Anfang, der die generelle Tauglichkeit der ABM für die kommunikationswissenschaftliche Forschung demonstrieren soll. So wurde die Isolationsfurchtschwelle in der einfachsten Interpretation von Mehrheit konstant für alle Agenten auf 50 Prozent festgesetzt. Zukünftig sollten Modellspezifikationen mit heterogenen Isolationsfurchtschwellen simuliert werden, um eine größere Annäherung an die Realität zu erreichen. So legen beispielsweise die mathematischen Simulationen von Granovetter und Soong (1988) nahe, dass die Systemdynamiken stark von diesen Bedingungen beeinflusst werden. Zudem sollten soziale Netzwerkstrukturen und Meinungsführerschaften in das Modell implementiert werden, um die Interaktionen zwischen den Agenten denen in realen Gesellschaften anzupassen (vgl. die Simulationsergebnisse bei Krassa, 1988). In den hier vorgestellten Modellen wirkt die Meinung jedes Agenten im Umfeld gleichermaßen auf die Wahrnehmung des Meinungsklimas. Agenten, die sich nicht im direkten Umfeld befinden, werden nicht berücksichtigt. Gerade für die Kombination solcher Konzepte, deren ganzheitliche empirische Beobachtung nahezu unmöglich ist, bietet die ABM neue Lösungsansätze. Aus den Simulationsergebnissen unter unterschiedlichen Spezifikationen können dann wiederum neue Einzelhypothesen über die Bedeutung wichtiger Variablen, wie z.B. der Meinungsverteilung im eigenen Netzwerk im Vergleich zur Gesamtbevölkerung empirisch untersucht werden. Schließlich sollten auch Versuche unternommen werden, Dynamiken in der veröffentlichten Meinung zu berücksichtigen. Hier sind sowohl empirische Inputparameter aus Inhalts- 
analysen wie auch komplexere Modellerweiterungen im Hinblick auf die massenmedialen Agenten denkbar.

\section{Chancen und Grenzen agentenbasierter Modelle in der Wirkungsforschung}

Dieser Beitrag hatte das Ziel, am Beispiel der Theorie der Schweigespirale zu verdeutlichen, wie ABM zur Erforschung gesellschaftlicher Medienwirkungen beitragen können. Besondere Potenziale liegen unserer Ansicht nach in der Entwicklung und Erweiterung von Theorien, die Bezüge zwischen Mikro- und Makroebene formulieren. Speziell bei Bottom-Up-Effekten stoßen auf Varianzzerlegung basierende empirische Zugänge an ihre Grenzen (Scheufele, 2008), und selbst theoretische Annahmen, wie sich diese Dynamiken von der Mikro- auf die Makroebene auswirken, sind nur schwierig präzise zu formulieren. Hier kann das aus der Komplexitätsforschung stammende Konzept der Emergenz (Holland, 1998, Sawyer, 2005), beobachtbar gemacht mit Hilfe von ABM, eine gute Hilfestellung bieten.

ABM ermöglichen die Durchführung virtueller Experimente, aus deren Simulation unter verschiedenen Randbedingungen sich konkrete Hypothesen über das Entstehen und den Ablauf der Emergenzen ableiten lassen (Epstein, 2006). Überall, wo die ganzheitliche empirische Erfassung von sozialen Kommunikations- und Medienwirkungsprozessen aus praktischen oder forschungsethischen Gründen nicht möglich ist, liegen die besonderen Reize der ABM. Individuenzentrierte Medienwirkungsansätze, netzwerkbasierte Konzepte zur interpersonalen Kommunikation und Meinungs- bzw. Informationsdiffusion sowie Theorien zur Bedeutung der Medienkommunikation für die Gesellschaft lassen sich in ABM miteinander verknüpfen und auf ihre Stimmigkeit hin explorieren.

Dabei sollte aber nie vergessen werden, dass es sich bei der ABM ausdrücklich nicht um eine empirische Methode handelt. Bereits Schnell (1990) hat - von der deutschen Fachöffentlichkeit leider weitgehend unbeachtet - vorgeschlagen, Computersimulationen zur Theoriebildung in den Sozialwissenschaften einzusetzen. Einen wesentlichen Grund für die Skepsis gegenüber dieser Methode sieht er in den komplexen empirischen Prognosemodellen der 1980er Jahre, deren Ergebnisse eine Scheingenauigkeit vermittelten, die sich meist nicht annähernd bewahrheite. Nach Schnell sind die Vorteile der ABM daher in der Theorieentwicklung und modifikation zu sehen. Die Computersprache der ABM zwingt Forscherinnen und Forscher zu einer hinreichenden Konkretisierung aller Annahmen. Die Simulation selbst kann die interne Konsistenz der Hypothesen innerhalb einer Theorie überprü- 
fen. Wenn die Simulationsergebnisse trotz einer korrekten Übersetzung in die Modellsprache keine theoriekonformen Ergebnisse liefern, so kann dies ein wichtiger Hinweis auf fehlende Theoriebestandteile oder eine Unstimmigkeit einzelner Hypothesen sein.

Ein solches Resultat darf jedoch nicht mit einer empirischen Falsifikation verwechselt werden. Es kann aber wertvolle Hinweise darauf geben, wo bei einer Erweiterung der Theorie anzusetzen ist. In diesem Sinne wollen wir dafür plädieren, die ABM reflektiert auf angemessene Probleme in der Medienwirkungsforschung einzusetzen - nicht als einen Ersatz, sondern vielmehr als eine sinnvolle Ergänzung des bestehenden Methodenrepertoires. 


\section{Literatur}

Boero, Riccardo / Squazzoni, Flaminio (2005): Does empirical embeddedness matter? Methodological issues on agent-based models for analytical social science. In: Journal of Artificial Societies and Social Simulation, Band 8, 6.

Bunge, Mario (2003): Philosophical dictionary. Amherst, MA: Prometheus.

Capra, Fritjof (1997): The web of life: A new synthesis of mind and matter. London, UK: Harper Collins.

Conte, Rosaria (2009): From simulation to theory (and backward). In: Squazzoni, Flaminio (Hrsg.) Epistemological aspects of computer simulation in the social sciences. Berlin: Springer.

Deffuant, Guillaume / Amblard, Frédéric / Weisbuch, Gérard / Faure, Thierry (2002): How can extremism prevail? A study based on the relative agreement interaction model. In: Journal of Artificial Societies and Social Simulation, Band 5, 1.

Donsbach, W. / Stevenson, R. L. (1986): Herausforderungen, Probleme und empirische Evidenzen der Theorie der Schweigespirale. In: Publizistik, Band 31, 7-34.

Entman, Robert M. / Herbst, Susan (2001): Reframing public opinion as we have known it. In: Bennett, W. Lance / Entman, Robert M. (Hrsg.) Mediated politics: Communication in the future of democracy. Cambridge, MA: Cambridge University Press.

Epstein, Joshua M. (Hrsg.): (2006) Generative social science: Studies in agent-based computational modeling. Princeton, NJ: Princeton University Press.

Esser, Frank (1993): Soziologie: Allgemeine Grundlagen. Frankfurt am Main: Campus.

Gilbert, Nigel (2007): Agent-based models. Thousand Oaks, CA: Sage.

Gilbert, Nigel / Troitzsch, Klaus G. (2005): Simulation for the social scientist, 2. Auflage. Maidenhead: Open University Press.

Gong, Xiaoguang / Xiao, Renbin (2007): Research on multi-agent simulation of epidemic news spread characteristics. In: Journal of Artificial Societies and Social Simulation, Band 10, 1.

González-Avella, Juan Carlos / Cosenza, Mario G. / Klemm, Konstantin / Eguíluz, Victor M. / San Miguel, Maxi (2007): Information feedback and mass media effects in cultural dynamics. In: Journal of Artificial Societies and Social Simulation, Band 10, 9.

Granovetter, Mark / Soong, Ronald (1988): Threshold models of diversity: Chinese restaurants, residential segregation, and the spiral of silence. In: Sociological Methodology, Band 18, 69-104.

Habermas, Jürgen (1995): Strukturwandel der Öffentlichkeit. Untersuchungen zu einer Kategorie der bürgerlichen Gesellschaft. Frankfurt am Main: Suhrkamp.

Hegselmann, Rainer / Krause, Ulrich (2002): Opinion dynamics and bounded confidence: Models, analysis and simulation. In: Journal of Artificial Societies and Social Simulation, Band 5, 2.

Holland, John H. (1998): Emergence: From chaos to order. Oxford, UK: Oxford University Press.

Jervis, Robert (1997): System effects: Complexity in political and social life. Princeton, NJ: Princeton University Press.

Kepplinger, Hans M. (1982): Die Grenzen des Wirkungsbegriffes. In: Publizistik, Band 27, 98-113.

Krassa, Michael A. (1988): Social groups, selective perception, and behavioral contagion in public opinion. In: Social Networks, Band 10, 109-136. 
Mainzer, Klaus (2007): Thinking in complexity: The computational dynamics of matter, mind, and mankind. Berlin: Springer.

McKeown, Gary / Sheehy, Noel (2006): Mass media and polarisation processes in the bounded confidence model of opinion dynamics. In: Journal of Artificial Societies and Social Simulation, Band 9, 11.

Miller, John H. / Page, Scott E. (2007): Complex adaptive systems: An introduction to computational models of social life. Princeton, NJ: Princeton University Press.

Mitchell, Melanie (2009): Complexity: A guided tour. New York, NY: Oxford University Press.

Noelle-Neumann, Elisabeth (1973): Kumulation, Konsonanz und Öffentlichkeitseffekt. Ein neuer Ansatz zur Analyse der Wirkung der Massenmedien. In: Publizistik, Band 18, $26-55$.

Noelle-Neumann, Elisabeth (1974): The spiral of silence: A theory of public opinion. In: Journal of Communication, Band 24, 43-51.

Noelle-Neumann, Elisabeth (1993): The spiral of silence: Public opinion, our social skin, 2. Auflage. Chicago, IL: University of Chicago Press.

Richter, Klaus / Rost, Jan-Michael (2004): Komplexe Systeme, 2. Auflage. Frankfurt am Main: Fischer.

Sawyer, Keith R. (2005): Social emergence: Societies as complex systems. Cambridge, UK: Cambridge University Press.

Schenk, Michael (2007): Medienwirkungsforschung, 3. Auflage. Tübingen: Mohr Siebeck.

Scherer, Helmut (1990): Massenmedien, Meinungsklima und Einstellung: eine Untersuchung zur Theorie der Schweigespirale. Wiesbaden: Westdeutscher Verlag.

Scheufele, Bertram (2008): Das Erklärungsdilemma der Medienwirkungsforschung: Eine Logik zur theoretischen und methodischen Modellierung von Medienwirkungen auf die Meso- und MakroEbene. In: Publizistik, Band 53, 339-361.

Schnell, Rainer (1990): Computersimulation und Theoriebildung in den Sozialwissenschaften. In: Kölner Zeitschrift für Soziologie und Sozialpsychologie, Band 42, 109-128.

Squazzoni, Flaminio (2008): The micro-macro link in social simulation. In: Sociologica, Band 1/2008.

Windrum, Paul / Fagiolo, Giorgio / Moneta, Alessio (2007): Empirical validation of agent-based models: Alternatives and prospects. In: Journal of Artificial Societies and Social Simulation, Band 10, 8 . 